Published in final edited form as:

Semin Cancer Biol. 2010 December ; 20(6): 370-376. doi:10.1016/j.semcancer.2010.09.003.

\title{
Molecular basis of CLL
}

Yuri Pekarsky $^{1}$, Nicola Zanesi ${ }^{1}$, and Carlo M. Croce ${ }^{1}$

${ }^{1}$ Human Cancer Genetics Program and Department of Molecular Virology, Immunology and Medical Genetics, OSU School of Medicine, Ohio State University, Columbus, Ohio

\begin{abstract}
B-cell chronic lymphocytic leukemia (CLL), the most common leukemia in the Western world, results from an expansion of a rare population of CD5+ mature B-lymphocytes. CLL occurs in two forms, aggressive and indolent. For the most part indolent CLL is characterized by low ZAP-70 expression and mutated IgH $\mathrm{V}_{\mathrm{H}}$; aggressive CLL shows high ZAP-70 expression and unmutated $\operatorname{IgH} \mathrm{V}_{\mathrm{H}}$. Although clinical features and genomic abnormalities in CLL have been studied extensively, molecular mechanisms underlying disease development are still emerging. In the last few years, several important insights were reported in this area. MiR-15/16 targeting BCL2 and $M C L 1$ and DLEU7 targeting TNF pathway were proposed as tumor suppressors at 13q14, a commonly deleted region in indolent CLL. Molecular details of how activation of TCL1, a critical oncogene in aggressive CLL, results in the initiation of this malignancy were clarified. Importance of these pathways was supported by investigations of several mouse models of CLL. Here, we present what has been learned from these new pathways, discuss mouse CLL models and how these mouse models recapitulate the molecular mechanisms of this common leukemia.
\end{abstract}

\section{Keywords}

CLL; TCL1; miR-15/16; DLEU7

\section{Introduction}

Chronic lymphocytic leukemia (CLL) is the most common human leukemia, accounting for $\sim 10,000$ new cases diagnosed each year in the United States and representing $30 \%$ of all leukemia cases [1]. CLL patients can often survive for a number of years showing relatively mild symptoms [1]. Indeed, a significant proportion of CLL cases are diagnosed incidentally. CLL leukemic cells usually show morphologically mature appearance and usually do not proliferate in vitro [1,2]. This disease occurs in two forms, aggressive and indolent, both forms are characterized by the clonal expansion of CD5 positive Blymphocytes [1,2]. For the most part aggressive CLL is characterized by high ZAP-70 expression and unmutated IgH $\mathrm{V}_{\mathrm{H}}$, while indolent CLL shows low ZAP-70 expression and mutated $\operatorname{IgH} \mathrm{V}_{\mathrm{H}}[1,2]$. For a number of years it was generally accepted that CLL is caused by an inherent defect in apoptosis, and that malignant lymphocytes accumulate due to diminished cell death [1,2]. Consistent with this theory, CLL cells are relatively inert, do not proliferate, and accumulate until levels not supportable by a patient. However, this original

Corresponding author: Yuri Pekarsky, Department of Molecular Virology, Immunology and Medical Genetics, Ohio State University, 460 W. $12^{\text {th }}$ Ave, BRT room 1090, Columbus, Ohio 43210, Tel: (614)2923120, Fax: (614)2924097, Pekarsky.Yuri@ osumc.edu.

Publisher's Disclaimer: This is a PDF file of an unedited manuscript that has been accepted for publication. As a service to our customers we are providing this early version of the manuscript. The manuscript will undergo copyediting, typesetting, and review of the resulting proof before it is published in its final citable form. Please note that during the production process errors may be discovered which could affect the content, and all legal disclaimers that apply to the journal pertain. 
view has been challenged in the last ten years. Several reports showed that high lymphocyte count in CLL patients is caused not only by the prolonged survival, but also by proliferating cells from the bone morrow, spleen or lymph nodes [3-5]. Immune incompetence is another important feature of CLL. Most patients develop severe hypogammaglobulinemia and impaired immune response to antigens [6].

Our understanding of molecular events and prognostic markers in CLL was significantly improved by using molecular genetic approaches such as PCR, fluorescence in situ hybridization and microarray analysis. Genomic aberrations were detected in over $80 \%$ of CLL cases. These common chromosomal abnormalities include 13q, 11q, 17p and 7q deletions, and trisomy 12 [7]. The 13q14 deletion represents the most common CLL aberration reported in $\sim 50 \%$ of all cases (mostly in indolent CLL), on the other hand, $\sim 10$ $20 \%$ of CLL cases do not show chromosomal aberrations [7].

\section{MiR-15/16 and DLEU7 at 13q14: a unique collaboration of coding and noncoding genes in indolent CLL}

MiR-15/16 at 13q14, discovery and function-Since 13q14 deletions also occur in mantle cell lymphoma, myeloma, and prostate cancer [1,2], it is possible that one or more tumor suppressor genes at 13q14 are important in the pathogenesis of CLL and other malignancies. To identify tumor suppressor gene (or genes) at 13q14 several groups have used positional cloning, and a region of more than $1 \mathrm{Mb}$ has been fully sequenced and characterized [8,9]. However, in spite of extensive research, none of the known genes of this region were found to be inactivated in CLL by deletions, mutations or promoter hypermethylation [8-11]. In order to finally find the elusive tumor suppressor gene at 13q14, in 2001 we generated somatic cell hybrids between mouse and CLLs cells carrying $13 q 14$ deletion and translocation. Using these hybrids we finally identified a 30-kb region of deletion between exons 2 and 5 of $L E U 2$ gene [12,13]. The translocation breakpoint was mapped in the same region [12]. Since $L E U 2$ had previously been excluded as a likely candidate tumor suppressor gene in CLL $[8,9,11,14]$ we continued to search for genes within the region and finally discovered that a cluster of two noncoding microRNA genes miR-15a and $m i R-16-1$, is located precisely within the deleted region ant at the translocation breakpoint [12]. Interestingly, this $m i R-15 a / m i R-16-1$ cluster is deleted or down-regulated in $\sim 66 \%$ of CLL cases [12]. In contrast, expression of other genes in the region such as DLEU1, DLEU2, RFP5 did not seem affected by $13 \mathrm{q} 14$ deletions $[8,9,13]$. To determine possible mechanism of $m i R-15 / 16$ tumor suppressor function, subsequent study examined expression levels of $m i R-15 / 16$ and $B C L 2$ (a predicted target of both, $m i R-15$ and $m i R-16$ ) in CLL [15]. These results showed that $m i R-15 a$ and $m i R-16-1$ expression is inversely correlated to $\mathrm{Bcl} 2$ expression in CLL and that both microRNAs negatively regulate $B C L 2$ at a posttranscriptional level [15]. Consequently, $B C L 2$ repression by these microRNAs induces apoptosis in a leukemic cell line [15], and $m i R-15 / 16$ showed a tumor suppressor function in vivo by inhibiting the growth of tumor engraftments of leukemic cells in nude mice [16]. These findings suggested that deletions of miR-15/16 result in Bcl2 up-regulation, contributing to the pathogenesis of CLL. The importance of $m i R-15 / 16$ was recently confirmed by a report studying CLL development in New Zealand black (NZB) mice [17]. Since these mice are susceptible to CLL, Raveche et al. carried out linkage analysis and discovered that mouse genomic region homologous to $13 \mathrm{q} 14$ is one of the loci associated with CLL. DNA sequencing identified a point mutation in miR-15/16 precursor and levels of $m i R-16$ were decreased in NZB lymphoid tissues [17].

DLEU7, a second tumor suppressor at 13q14-Recently, a high-resolution map of $13 q 14$ deletions using 171 CLL samples was reported [18]. These study used microarray based technology to determine the minimal deleted region at 13q14 in CLL. Interestingly, in 
addition to $m i R-15 / 16$, this region also contained $D L E U 7$ gene located telometic to miR-15/16 [18], and previously identified as a candidate tumor suppressor gene at $13 \mathrm{q} 14$ [19]. DLEU7 encodes a 221 amino acid protein with no homology to known proteins. Sequencing of DLEU7 coding exons failed to find mutations in 45 CLL samples, although DLEU7 promoter was methylated in $61 \%$ of CLL [19]. Since DLEU7 is the only protein coding gene located within the minimal deleted region at $13 \mathrm{q} 14$, very recently we investigated whether DLEU7 can function as a tumor suppressor and cooperate with miR-15/16 [20]. We first examined expression of DLEU7 in CLL samples and normal CD19+ B-cells by real time RT-PCR results. All CLL samples examined showed decreased DLEU7 expression, while over $50 \%$ showed decrease of ten fold or more. Expression of both, $m i R-15$ and $m i R-16$, was also decreased in over $90 \%$ of CLL samples examined [20]. These results were consistent with previous study showing that $D L E U 7$ is methylated and in essence is not expressed in CLL [19].

Recent studies of CLL animal models as well as mechanistic studies demonstrated the importance of the NF-kB pathway in the pathogenesis of CLL CLL (reviewed in [21]). For instance, transgenic expression of a proliferation-inducing TNF ligand (APRIL), a member of the tumor necrosis factor (TNF) superfamily involved in NF-kB activation resulted in the development of a disease very similar to CLL (see below) [22]. In our recent report we found that Tcl1 activation caused CLL by activation of NF-kB pathway and inhibition of AP-1 [23]. Since these studies suggested a significant role for the NF-kB pathway in the pathogenesis of CLL [21], we investigated whether Dleu7 might function as an inhibitor of $\mathrm{NF}-\mathrm{kB}$, in particular in the pathway involving APRIL.

APRIL and its close relative BAFF are recent members of TNF superfamily expressed almost exclusively in lymphoid cells [24]. Interestingly, both BAFF and APRIL show elevated expression levels in various B-cell malignancies including diffuse large cell lymphoma, mantle cell lymphoma and CLL $[21,24]$. APRIL binds with high affinity to two receptors, BCMA (B-cell maturation antigen) and TACI, both are members of TNF receptor superfamily (Figure 1) [24]. BCMA is mostly expressed in mature B- and T-cells, while TACI is detected in activated T-cells and subpopulations of B-cells [24]. They stimulate NF$\mathrm{kB}$ pathway by interaction with various TRAFs [24]. Thus, NF-kB activation through TACI and BCMA could be important in the pathogenesis of CLL [21]. Since DLEU7 is located within the 13q14 deleted region we investigated whether its expression has an effect on NF$\mathrm{kB}$ activation by TACI and BCMA. Transfection experiments confirmed our hypothesis: Dleu7 expression inhibited NF-kB activation by BCMA over 5 fold, similarly, NF-kB activation by TACI was inhibited over 4 fold [20]. Thus, we demonstrated that Dleu7 functions as NF-kB inhibitor in a pathway critical for the pathogenesis of CLL (Figure 1).

Nuclear factor of activated T-cells (NFAT) can also be activated by TACI and BCMA, same molecules responsible for NF-kB activation (Figure 1) [25]. Previous reports showed that nuclear activated NFAT is a hallmark of unstimulated CLL cells and that NFAT is a critical factor for CD5 expression in B-cells [26,27]. Since Dleu7 inhibits NF-kB activation by TACI and BCMA, we hypothesized that Dleu7 can also inhibit the ability of TACI and BCMA to induce NFAT mediated transactivation. Indeed, we showed that Dleu7 expression inhibited this activation $\sim 8$ fold and concluded that Dleu7 functions as NFAT inhibitor [20]. Further co-immunoprecipitation experiments confirmed that Dleu7 functions as TACI and BCMA inhibitor by direct association with these molecules [20]. Although these data strongly indicate that Dleu7 might function as a tumor suppressor in CLL it is important to show this function in cellular assays. To show that this Dleu7 can indeed function a tumor suppressor we used adenovirus expressing Dleu7 and GFP, as a control. These experiments showed that Dleu7 expression results in $>2$ fold decrease of cells in the $S$ phase and in $>3$ fold induction in apoptosis as measured by Annexin V incorporation [20]. All these data 
clearly indicate we identified $D L E U 7$ as a second tumor suppressor in $13 \mathrm{q} 14$ in addition to miR-15/16.

In summary, recent investigations determined that $13 \mathrm{q} 14$ contains two cooperating tumor suppressors, miR-15/16 and DLEU7. miR-15/16 deletions result a constitutive increase of Bcl 2 expression, DLEU7 inactivation causes induction of TNF signaling through TRAFs. Intriguingly, these two events cause CLL phenotype closely resembling oncogenic events in TRAF2DN/BCL2 transgenic mouse model (see below) [20].

\section{TCL1, a critical oncogene in aggressive CLL}

Activation of the TCLI oncogene is arguably one of most studied initiating events in the pathogenesis of aggressive CLL. TCL1 (T cell leukemia/lymphoma 1) was first identified as a target of translocations and inversions at $14 \mathrm{q} 32.1$, common chromosomal aberrations in Tcell prolymphocytic leukemias [28]. Expression analyses of TCL1 revealed that this gene is mostly expressed in B-cells and to a much lesser extent in T-cells [28]. In B-cells, Tcl1 is expressed in all stages of development, except mature B-cells [29,30]. Since our study demonstrating that TCL1 deregulation in mouse B-cells results in aggressive CLL [31], several additional reports have indicated that Tcl1 is critical molecule in the CLL pathogenesis [13,32]. Importantly, high Tcl1 expression in human CLL correlates with aggressive CLL phenotype showing unmutated immunoglobulin variable region genes and ZAP70 positivity [32].

TCL1 function in CLL-The molecular mechanism of Tcl1 mediated oncogenesis was not known untill ten years ago when we and others reported that Tcll functions as an activator PI3K - Akt(PKB) oncogenic pathway [33,34]. Akt kinase encoded by the $A K T$ oncogene plays an important role in the regulation of a number of signaling pathways involved in cell proliferation, survival, and death in numerous cell types including B- and Tcells [35]. Our results showed that Tcl1 and Akt physically interact and that PH domain of Akt is responsible for this binding [33]. Using in vitro and in vivo kinase assays we showed that Akt bound to Tcl1 exhibits significant increase in the kinase activity [33,34]. We also demonstrated that Tcl1 mediates the nuclear translocation of Akt [33].

More recently we investigated whether TCL1 expression in CLL is regulated by microRNAs [36]. Since Tcl1 is up-regulated in aggressive CLL, we were interested in microRNAs down-regulated in aggressive CLL that potentially target TCL1. These experiments revealed that expression of $m i R-29 b$ and $m i R-181 b$ significantly inhibits Tcl1 expression and that $m i R-29 b$ and $m i R-181 b$ target $T C L 1$ expression at mRNA and protein levels [36]. Intriguingly, a microRNA signature was previously reported with 13 microRNAs that differentiate aggressive and indolent CLL [37,38]. In this signature only four microRNAs were down-regulated in aggressive CLL versus indolent CLL. Remarkably, of the four, three are isoforms of $m i R-29(m i R-29 a-2, m i R-29 b-2$ and $m i R-29 c)$ [36]. This confirms the importance of the interaction between $m i R-29$ and TCL1 in aggressive CLL.

Is involvement of Tcl1 in Akt activation important in the pathogenesis of CLL? The fact that transgenic mice overexpressing activated Akt in T-cells develop T-cell leukemias [39] provides an important evidence that Akt contributes to lymphomagenesis in T-cells initiated by TCL1 activation (Figure 2). On the other hand, transgenic mice expressing activated Akt in B-cells do not develop lymphoid malignancies (YP and CMC, unpublished results), and conditional Pten knockout in mouse B-cells expressing constitutively active Akt did not cause B-cell transformation [40]. Thus, it is likely that Tcl1 mediated CLL pathogenesis is caused by mechanism(s) other than Akt activation. 
In 2008 we were able to uncover such mechanism [23]. We hypothesized that some CLL samples might have gain of function mutations in TCL1, and that functional analysis of these mutations could point to molecular mechanisms of Tcll mediated B-cell transformation. Thus, we sequenced the TCL1 gene in 600 CLL samples and identified two heterozygous mutations resulting in amino acid substitutions, T38I and R52H. Interestingly, RT-PCR results showed that the T38I mutant TCL1 mRNA was the major expressed allele in the CLL of origin, accounting for $\sim 80 \%$ of the TCL1 mRNA, and the R52H allele was the only allele expressed [23]. Since NF-kB activation is critical in CLL development, we first examined if $T C L 1$ mutants show gain of function in NF-kB activation. Using reported system we showed that Tcl1 activated NF-kB activity $2-4$ fold by a mechanism independent of Akt. However, in these experiments the Tcl1 mutants did not exhibit gain of function in the activation of NF-kB [23].

After examining several other pathways we focused on activator protein 1 (AP-1) dependent transcription [41]. The classical AP-1 complex contains c-Jun and c-Fos, although other Jun proteins (JunB and JunD) and Fos proteins (Fra-1, Fra-2 and FosB) have also been reported [42]. Several studies reported that AP-1 induces apoptosis by activating pro-apoptotic genes [43-45]. For example, activation of AP-1 was reported in lymphoid cells after serum deprivation and this induction was associated with apoptosis [46]. Also, mouse knockout studies showed that JunB deficiency caused induction of B-cell leukemias [47]. Thus, we investigated whether Tcl1 can inhibit AP1-dependent transcription. Using reported system we showed that Tcl1 expression inhibited AP-1 dependent transactivation $\sim 2.5$-fold while Tcl1 T38I caused a dramatic $\sim 100$-fold inhibition, and the R52H mutant showed $\sim 4$ fold inhibition [23]. Similar results were obtained using individual components of the AP-1 complex, such as c-Jun, c-Fos and JunB. In all these cases Tc11 T38I mutant inhibited more potently than WT Tcl1 strongly indicating that Tcl1 mutants show gain of function effect in AP-1 inhibition [23]. To elucidate the mechanism of this inhibition we carried out a series of co-immunoprecipitation experiments. These results revealed that Tcl1 specifically interacts with c-Jun, c-Fos and JunB. Thus, we concluded that Tcl1 physically interacts with AP-1 components and functions as an AP-1 inhibitor [23]. The fact that both Tcl1 mutants identified in CLL patients show gain of function properties in this pathway suggests the ability of Tcl1 to inhibit AP-1 dependent transcription is critical in the pathogenesis of CLL (Figure 2).

In summary, in the last ten years we discovered two critical pathways involving Tcl1 (Figure 2). In T-cells, Tcl1 activates Akt, this leads to increased proliferation, inhibition of apoptosis and T-cell transformation [33]. In B-cells, Tcl1 functions as transcriptional regulator, it activates NF-kB and inhibits AP-1 [23]. This leads to defects in apoptosis, increase in cell survival, and CLL pathogenesis.

\section{Mouse models of CLL}

Although dysregulation of a specific pathway (or gene) in CLL suggests possible involvement in this disease, the final proof of the critical role of this pathway (or gene) in the pathogenesis of CLL requires generation of animal models showing CLL phenotype. In this chapter we discuss animal models of CLL which confirmed the importance of molecular mechanisms we have described above.

TCL1 driven CLL mouse model-Several years ago, we produced transgenic mice in which the expression of $T C L 1$ was under the control of a $\mathrm{V}_{\mathrm{H}}$ promoter- $\operatorname{Ig}_{\mathrm{H}}-\mathrm{E} \mu$ enhancer that targets expression of the transgene to immature and mature B-cells [31]. The immunophenotypic profile of peripheral blood lymphocytes (PBLs) from these mice between one and nine months of age detected an expansion of the $\mathrm{B} 220 / \mathrm{IgM}^{+}$population, in particular 
those that were $\mathrm{CD}^{+} / \mathrm{B} 1$, whereas such cells are generally infrequent [48]. This expansion was revealed in $100 \%$ of the transgenic mice by six months of age, without sign of clinical disease [31]. Fluorescence-activated cell sorting (FACS) analysis detected a phenotypically homogeneous $\mathrm{CD}^{+} \mathrm{IgM}^{+}$population significantly expanded in the peritoneal cavity of the transgenic mice starting at two months of age (44\%) that became then evident in spleen (9\%) by four months and bone marrow by eight months (43\%) [21,31]. At this age, E $\mu$ $T C L 1$ mice presented a slightly enlarged spleen and a much higher cellularity in the peritoneal cavity, ranging between 50- to 100-fold increase. The cell cycle distribution and the rate of cell proliferation in spleen and peritoneal cavity of these transgenic mice were also investigated and we found that $\mathrm{IgM}^{+} \mathrm{CD}^{+}$sorted populations were arrested in the $\mathrm{G}_{0} /$ $\mathrm{G}_{1}$ phase of the cell cycle and did not actively divide. The onset of a frank leukemia in the elderly mice provided the final evidence of the establishment of a murine model for human CLL [21,31]. All mice between 10 and 20 months of age became visibly ill and presented with spleno- and hepatomegaly associated with high counts of white blood cells (WBCs). Moreover, some mice also developed advanced lympho-adenopathy, another hallmark of human CLL [21,31]. Simultaneously, another Tcl1 transgenic mouse model was created to express Tcl1 in both, B- and T-cells [49]. The phenotype of CLL like disease in this second mouse was similar to that described for our Tcl1 transgenics.

Later on, we studied the spontaneous tumor phenotype of E $\mu-T C L 1$ transgenic mice more in detail [50]. An extensive pathologic analysis of these transgenic animals revealed that, in addition to CLL leukemias, more than $30 \%$ of mice showed the onset of solid malignant tumors, even though the tumors themselves lacked Tcl1 expression. Secondary malignancies are also frequent complications in patients with CLL [51] and they are the most common cause of death in these patients [52]. Higher risk of secondary non-hematological neoplasms in patients with CLL has several explanations including genetic predisposition, immune deficiency, carcinogen exposure, and the side-effects of therapeutic treatments [53]. In our CLL model, $25 \%$ of secondary cancers were malignant pilomatrixoma, a type of skin cancer extremely rare in mice. Thus far, the E $\mu-T C L 1$ model seems to be the only one reported that displays the onset of secondary malignancies in CLL-prone mice [21,50].

The homogeneity of the genetic background of a mouse model is an important factor to evaluate the effects of novel therapies. For this reason, we established CLL transplants in syngeneic mice that allowed us at first to propagate, and later to therapeutically treat, the same leukemia in different mice but with the same genetic background. Such a system allows for the study of each leukemia and is a necessary step on the road to find drugs that specifically interfere with the pathways involved in the disease [21,50]. To determine whether the development of mouse CLL is dependent on the Akt pathway, we investigated whether the disruption of Akt signaling through mTOR, by the mTOR inhibitor rapamycin, prevented or cured mouse CLL. The treatment prolonged the life of all treated animals in either the prevention or the therapeutic group. Mammalian TOR normally regulates translation by regulation key components of the protein synthesis machinery, including ribosomal protein S6 kinase. We found that lymph nodes of untreated mice expressed phosphorylated S6, whereas lymphoid tissues from rapamycin-treated mice did not show any phosphorylation. In conclusion, rapamycin inhibited mTOR activity in the Tcl1dependent CLL mouse model [50].

As we reported in the previous chapter, levels of TCL1 expression in CLL are regulated by miR-29 and mir-181 that target $3^{\prime}$ UTR of TCL1. To determine whether treatment with microRNAs targeting TCL1 can inhibit CLL in mice, we designed TCL1 transgenic mice using a construct containing the $3^{\prime}$ and $5^{\prime}$ UTRs of TCL1 under B cell - specific E $\mu$ promoter (E $\mu$-TCL1 Full Length). At the age of 16-20 months, these mice showed a CLL-like disease [54]. Immunophenotyping revealed accumulation of CD5+ CD23+ B220+ population in 
spleens and lymph nodes. Our findings showed that CD5+ CD23+ B cell populations from $\mathrm{E} \mu-T C L 1 \mathrm{FL}$ mice actively proliferated and showed significantly increased levels of

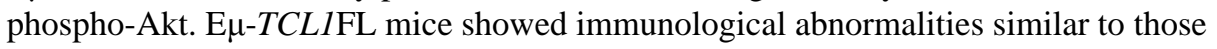
present in human CLL, including hypoimmunoglobulinemia, abnormal levels of cytokines and impaired immune response. Overall, these results revealed biochemical and immunological similarities to Tcl1 driven CLL mouse models and human CLL [54].

In summary, a vast corpus of information has been collected in the last eight years about leukemia developed by the $\mathrm{E} \mu-T C L 1$ transgenic mice, demonstrating its clear similarity to human CLL.

MiR-29 driven mouse model of CLL-We recently found that $m i R-29$ is overexpressed in indolent human CLL, compared to aggressive CLL and normal CD19+ B-cells. To elucidate the role of $m i R-29$ in B-cell leukemias we created transgenic mice overexpressing miR-29 in B-cells and recently we reported the phenotype of this new mouse model [55]. Immunophenotypic profile of spleen lymphocytes from $m i R$ - 29 transgenics showed increased populations of CD5+CD19+IgM+ B-cells, a typical feature of CLL. Between the age of 12 and 24 months a markedly expanded $\mathrm{CD}^{+} \mathrm{B}$-cell population was evident in spleens of 34 of $40(85 \%)$ miR-29 transgenic mice; $50 \%$ of B-cells in these transgenics were CD5 positive [55]. Interestingly, of $20 \mathrm{miR}-29$ transgenic mice followed to 24-26 months of age, only 4 (20\%) developed frank leukemia and died of the disease. Since almost all miR-29 transgenics showed expanded CD5+CD19+IgM+ B-cell populations, but only $20 \%$ developed a frank leukemia, we concluded that $m i R-29$ transgenics developed a disease similar to indolent CLL [55]. Moreover, $m i R$-29 mice showed significant increases in $\%$ of leukemic cells with age. In mice younger than 15 months, CD5+ leukemic cells were only $\sim 20 \%$ of total B-cells; on the other hand, at the age of $20-26$ months, $>65 \%$ of all B-cells were CD5+. These results showed a gradual progression of indolent CLL in miR-29 transgenics [55]. To determine whether leukemic cells from miR-29 mice divide, we did measure the proliferative capacity of CD5+ leukemic B-cells compared to WT CD19+ spleen lymphocytes. BrdU incorporation experiments showed significant proliferation in miR-29 transgenic B-cells, while no proliferation was found in CD19+ WT lymphocytes [55]. These results suggest that $m i R-29$ over-expression plays an important role in promoting B-cell proliferation. Because progressive hypogammaglobulinemia and immune incompetence are important features of human CLL [6], we analyzed serum levels of immunoglobulins and the immune response to SRBC antigen in the serum of miR-29 mice and wild type littermates. We showed that in $m i R-29$ transgenics immune response to SRBC antigen and serum levels of immunoglobulins were significantly decreased [55]. Thus, these findings confirmed our initial observations that $m i R-29$ transgenics develop a disease mimicking the indolent human counterpart.

MiR-15a/16-1 cluster knockout mouse model of CLL-As recently reviewed [56], the literature on the importance of miRNAs deregulation in creation of mouse models of lymphocytic leukemias is becoming increasingly richer. Early this year, Dalla-Favera and colleagues published the genetic validation that deletion of miR-15a/l6-1 leads to CLL in mice [57]. In order to identify the genetic elements targeted by the putative 13q14 tumor suppressor and to determine their contribution to the pathogenesis of CLL, they generated knockout mice that carried conditional alleles that either mimicked the deletion of the minimal deleted region (MDR), previously described in human CLL $[9,58]$ and comprising the entire Dleu2 gene, or that specifically deleted the miR-15a/16-1 cluster without affecting the expression of Dleu2. At the age of 2-4 months, homozygous Mdr-/- and miR-15a/ 16-1-/- mice showed normal percentages of $\mathrm{B}$ and $\mathrm{T}$ cell subpopulations and normal development of lymphoid organs. However, one year old $M d r-/-$ mice showed higher percentages of CD5+B220 B cells among mononuclear cells in the peritoneal cavity (50\% 
vs. $15 \%$ in controls). Similar observations were made in miR-15a/l6-1-/-rodents. Thus, deletions of the $M d r$ and $m i R-15 a / 16-1$ are associated with expansion of CD5+B220 cells in the peritoneal cavity. In a fraction of the animals, clonal CD5+ B cell populations were accompanied by significant infiltration of lymphoid organs with histopathologic features resembling human CLL $[56,57]$. These mice displayed an enlargement of the spleen white pulp due to the expansion or accumulation of small lymphocytes with a pattern similar to CLL. Discrete aggregates of small lymphocytes were also observed in the bone marrow. Overall, $27 \%$ of $M d r-/-$ and $21 \%$ of $m i R-15 a / 16-1-/-$ mice developed CLL. Altogether $42 \%$ of $M d r-/-$ and $26 \%$ of miR-15a/16-1-/- mice, 15-18 months of age, developed some type of clonal B cell lymphoproliferation. $M d r-/-$ mice also showed a trend toward developing B cell tumors (24\%), suggesting that monoallelic deletion of the $M d r$ can cause disease. This trend is not evident in $m i R-15 a / 16-1$-deleted mice, probably due to the lower penetrance of the phenotype. $M d r-/-$ mice died earlier than their wild type littermates, suggesting that the homozygous mice eventually succumb to their tumors at an advanced age [56,57]. On the contrary, the survival of $m i R-15 a / 16-1-/-$ mice was not significantly different from wild type littermates, suggesting that these mice show a milder disease course than their $M d r$ counterparts. In conclusion, both $M d r-/-$ and $m i R-15 a / 16-1-/-$ mice developed lymphoproliferations with an indolent disease course reminiscent of human CLL, with $M d r-/-$ mice displaying a more aggressive phenotype. The significantly more aggressive phenotype displayed by $M d r-/-$ mice suggests that additional genetic elements within the MDR locus may contribute to the tumor suppressive function. One candidate for such a role is the DLEU2 gene itself. Besides providing the primary transcript for the production of $m i R-15 a / 16-1$, it produces a spliced cytoplasmic RNA that may have important regulatory functions not yet detected [56,57].

To understand the mechanism by which the deletions lead to clonal B lymphoproliferations, Klein et al. [57] investigated whether miR-15a/l6-1 deletions could affect proliferation of mouse $\mathrm{B}$ cells. BrdU incorporation assays, by measuring active DNA-synthesis, demonstrated that $m i R-15 a / 16-1-/-\mathrm{B}$ cells begin to synthesize DNA earlier than wild type B cells. Mitogen-stimulated B cells purified from miR-15a/16-1-/- or $M d r-/-$ and wild type mice were analyzed for levels of phosphorylated retinoblastoma $(\mathrm{p}-\mathrm{Rb})$ protein, an indicator of entry into the cell cycle. P-Rb could be revealed at earlier time points in both miR-15a/16-1-/- and $M d r-/-$ compared with wild type B cells. Consistently, the expression of the cell cycle inhibitory protein cyclin-dependent kinase inhibitor p27-Kip1 appeared to follow similar kinetics. To unravel the individual contributions of DLEU2 versus the $m i R-15 a / 16-1$ cluster to the lymphoproliferative phenotype, the authors created an inducible in vitro system in which the DLEU2 transcript and the miR-15a/16-1 cluster were separately re-expressed in the human I83E95 cell line that was derived from a 13q14-/CLL. The results showed that miR-15a/16-1 expressing cells, but not those expressing DLEU2, were impaired in proliferation. In accord with this observation, miR-15a/16-1 expressing cells had a higher fraction of cells in the G0/G1 phase compared with both $D L E U 2$ and the control, as documented by BrdU incorporation assays. Thus, expression of the miR-15a/16-1 cluster seems to control cellular proliferation, possibly by inhibiting the G0/G1 phase transition. Altogether, the results of the knockout and re-expression experiments suggest that the miR-15a/l6-1 cluster exhibits negative control of proliferation both in human and mouse B cells. Conversely, the DLEU2 transcript per se did not affect proliferation of 13q14 homozygous deleted cells [56,57]. Because their findings suggested that miR-15a/16-1 may affect the G0/G1-S phase transition in B cells, the authors sought to identify candidate G0/G1-S phase-related genes among inferred miR-15a/l6-1 targets predicted by different computational target prediction algorithms. Seven proteins (cyclins Ccnd2, Ccnd3, and Ccne1, and cyclin-dependent kinases Cdk4, Cdk6, Chk1, and Mcm5) known to be involved in the regulation of the G0/G1-S phase transition, were downregulated in I83E95 13q14-/- cells upon miR-15a/l6-1 expression. Similarly, the same gene products 
showed higher expression levels in anti-IgM-stimulated $m i R$-15a/16-1-deficient mouse B cells. Though it remains to be determined whether the proliferation-associated genes identified represent direct or indirect miR-15a/16-1 targets, the concurrent results obtained in two independent genetically defined $m i R-15 a / 16-1$ knockout systems strongly indicate that these miRNAs regulate the expression of multiple genes involved in the G0/G1-S phase transition in both human and mouse B cells [56,57].

BCL2 and TRAF2 transgenic mouse model of CLL-As mentioned previously in this review, a link between genomic abnormalities and high expression levels of $\mathrm{Bcl} 2$ in the majority of CLL patients was established by the discovery of the role of miR-15a/16-1 in the disease. Thus, deregulation of $B C L 2$ is most likely a major contributor to the pathogenesis of CLL, specifically the indolent form of CLL that is often accompanied by 13q14 deletions. A study by Dr. Reed's laboratory provided an important evidence in favor of this hypothesis [59]. The study was based on two previous models, a mouse over-expressing Bcl2 in the lymphoid system [60] and a transgenic line over-expressing an isoform of TRAF2 in murine B- and T-cells [61]. TNF receptor associated factor 2 (TRAF2) belongs to a family of adaptor proteins that bind to TNF receptor family members and mediate the activation of NF-kB and JNK by the tumor necrosis factor proteins (Fig. 2) [62]. Increase in lymphocyte proliferation and survival is led by TNF-mediated signaling [24]. TRAF2 transgenic mice over-expressed TRAF2 mutant, lacking the N-terminal RING and zinc finger domains located at the N- terminal of the molecule $(T R A F 2 D N)$, which mimics TRAF1 [21,61]. The mice developed splenomegaly, lympho-adenopathy and a higher number of B-cells but failed to develop a clear blood neoplasia. $B C L 2$ transgenics were generated by using a construct that mimics $t(14 ; 18)$ translocation juxtaposing $B C L 2$ oncogene with the immunoglobulin heavy-chain locus at $14 \mathrm{q} 32$ observed in human follicular lymphomas. These mice did not develop any malignant phenotype, but displayed polyclonal expansions of B-cells and a more pronounced in vitro B-cell survival [21,60]. Contrary to the single transgenic animals, TRAF2DN/BCL2 double transgenics developed severe splenomegaly, and most rodents developed a B-cell leukemia similar to CLL with B-cell blood count as high as 40 times normal [59]. The mice died prematurely at 6-14 months of age, though Bcl2 or TRAF2DN single transgenics had a regular lifespan. As in the case of the TCL1 transgenic mice, malignant cells were $\mathrm{B} 220^{+} \mathrm{CD}^{+}$, with a small number of animals having B-cell expansions of a different subtype. In conclusion, it is still uncertain if the TRAF2DN/ $B C L 2$-driven leukemia resembles an indolent or an aggressive form of CLL [21].

APRIL driven mouse model of CLL-NF-kB is a downstream target of TRAFs (Figure 1). Hence, it is possible that activation of NF-kB plays a role in the pathogenesis of CLL. Thus, a study described another mouse model for CLL suggesting the involvement of NF$\mathrm{kB}$. The study reported a transgenic line expressing APRIL (a proliferation-inducing ligand) under control of the T-cell specific lck promoter [22]. APRIL is a secreted protein, thus its over-expression in T-cells caused elevated APRIL levels in serum. In fact, APRIL works systemically in these transgenic mice affecting proliferation and survival of B-cells [22]. These expanded mature B-cells revealed significant increase in survival in vitro in comparison to normal B-lymphocytes [21,22]. The expansion of $\mathrm{B} 220^{+} \mathrm{CD} 5^{+}$cells was observed in $\sim 40 \%$ of transgenic animals in contrast with other described CLL mouse models where the penetrance was appoximately $100 \%$. Generally, APRIL transgenics showed much milder phenotype than TCL1 or TRAF2DN/BCL2 transgenic animals. APRIL mice showed only mild expansion of splenic B-cells, they did not develop high blood white cells count and did not die prematurely from leukemia or lymphoma. Hence, it is possible that APRIL predisposes CD5+ B-cells to malignancy, but this occurrence would require also a second hit, such as Bcl2 or Tcl1 up-regulation. 


\section{Conclusions}

In the last few years, several important studies uncovering molecular mechanisms of CLL pathogenesis were reported. In addition to miR-15/16 targeting BCL2, DLEU7 was identified as second tumor suppressor at 13q14 [20]. Also, we demonstrated that Tcl1 functions as a transcriptional regulator in the pathogenesis of aggressive CLL [23]. Importance of $m i R-15 / 16$ and TCL1 in CLL was supported by generating TCL1 transgenic and miR-15/16 knockout mouse models, both showing CLL phenotype. Activation of NF-kB pathway has evolved as another critical event in CLL pathogenesis since NF-kB is activated in three CLL mouse models, APRIL driven, BCL2 and TRAF2 driven, and TCL1 driven (Tcl1 functions as NF-kB activator) (Figure 1).

$B C L 2 / T R A F 2 D N$ transgenic mouse model represents a very interesting parallel and confirmation for the idea of two cooperating tumor suppressors at 13q14. Although $B C L 2$ or TRAF2DN single transgenics do not develop CLL, TRAF2DN/BCL2 double transgenic mice over time developed B-cell leukemias of B220+CD5+ phenotype similar to CLL.[59] Since human 13q14 region contains two cooperating tumor suppressors, miR-15/16 and DLEU7, $13 q 14$ deletions cause inactivation of both genes. MiR-15/16 inactivation causes a constitutive increase of $\mathrm{Bcl} 2$ expression, DLEU7 inactivation causes induction of TNF signaling through TRAFs. These two events cause the CLL phenotype closely resembling the oncogenic events in TRAF2DN/BCL2 transgenic mouse model (Figure 1) [20].

\section{Acknowledgments}

This work was supported by an ACS Research Scholar grant (Y. Pekarsky) and by NCI grants (CMC).

\section{References}

1. Sgambati, M.; Linet, M.; Devesa, S. Chronic Lymphocytic Leukemia, Epidemiological, Familial, and Genetic Aspects. In: Cheson, Bruce, editor. Chronic Lymphocytic Leukemias. 2. Marcel Dekker, Inc; New York: 2001. p. 33-62.Revised and Expanded

2. Bullrich, F.; Croce, C. Molecular Biology of Chronic Lymphocytic Leukemia. In: Cheson, Bruce, editor. Chronic Lymphocytic Leukemias. 2. Marcel Dekker, Inc; New York: 2001. p. 9-32.Revised and Expanded

3. Chiorazzi N. Cell proliferation and death: forgotten features of chronic lymphocytic leukemia B cells. Best Pract Res Clin Haematol 2007;20:399-413. [PubMed: 17707829]

4. Messmer BT, Messmer D, Allen SL, Kolitz JE, Kudalkar P, Cesar D, et al. In vivo measurements document the dynamic cellular kinetics of chronic lymphocytic leukemia B cells. J Clin Invest 2005;115:755-64. [PubMed: 15711642]

5. Sieklucka M, Pozarowski P, Bojarska-Junak A, Hus I, Dmoszynska A, Rolinski J. Apoptosis in BCLL: the relationship between higher ex vivo spontaneous apoptosis before treatment in III-IV Rai stage patients and poor outcome. Oncol Rep 2008;19:1611-20. [PubMed: 18497973]

6. Caligaris-Cappio F, Hamblin TJ. B-cell chronic lymphocytic leukemia: a bird of a different feather. J Clin Oncol 1999;17:399-408. [PubMed: 10458259]

7. Dohner H, Stilgenbauer S, Benner A, Leupolt E, Krober A, Bullinger L, et al. Genomic aberrations and survival in chronic lymphocytic leukemia. N Engl J Med 2000;343:1910-6. [PubMed: 11136261]

8. Bullrich F, Fujii H, Calin G, Mabuchi H, Negrini M, Pekarsky Y, et al. Characterization of the 13q14 tumor suppressor locus in CLL: identification of ALT1, an alternative splice variant of the LEU2 gene. Cancer Res 2001;61:6640-8. [PubMed: 11559527]

9. Migliazza A, Bosch F, Komatsu H, Cayanis E, Martinotti S, Toniato E, et al. Nucleotide sequence, transcription map, and mutation analysis of the 13q14 chromosomal region deleted in B-cell chronic lymphocytic leukemia. Blood 2001;97:2098-104. [PubMed: 11264177] 
10. Rondeau G, Moreau I, Bezieau S, Petit JL, Heilig R, Fernandez S, et al. Comprehensive analysis of a large genomic sequence at the putative B-cell chronic lymphocytic leukaemia (B-CLL) tumour suppresser gene locus. Mutat Res 2001;458:55-70. [PubMed: 11691637]

11. Mertens D, Wolf S, Schroeter P, Schaffner C, Dohner H, Stilgenbauer S, et al. Down-regulation of candidate tumor suppressor genes within chromosome band $13 q 14.3$ is independent of the DNA methylation pattern in B-cell chronic lymphocytic leukemia. Blood 2002;99:4116-21. [PubMed: 12010815]

12. Calin GA, Dumitru CD, Shimizu M, Bichi R, Zupo S, Noch E, et al. Frequent deletions and downregulation of micro- RNA genes miR15 and miR16 at 13q14 in chronic lymphocytic leukemia. Proc Natl Acad Sci U S A 2002;99:15524-9. [PubMed: 12434020]

13. Pekarsky Y, Calin GA, Aqeilan R. Chronic lymphocytic leukemia: molecular genetics and animal models. Curr Top Microbiol Immunol 2005;294:51-70. [PubMed: 16323427]

14. Wolf S, Mertens D, Schaffner C, Korz C, Dohner H, Stilgenbauer S, et al. B-cell neoplasia associated gene with multiple splicing (BCMS): the candidate B-CLL gene on 13q14 comprises more than $560 \mathrm{~kb}$ covering all critical regions. Hum Mol Genet 2001;10:1275-85. [PubMed: 11406609]

15. Cimmino A, Calin GA, Fabbri M, Iorio MV, Ferracin M, Shimizu M, et al. miR-15 and miR-16 induce apoptosis by targeting BCL2. Proc Natl Acad Sci U S A 2005;102:13944-9. [PubMed: 16166262]

16. Calin GA, Cimmino A, Fabbri M, Ferracin M, Wojcik SE, Shimizu M, et al. MiR-15a and miR-16-1 cluster functions in human leukemia. Proc Natl Acad Sci U S A 2008;105:5166-71. [PubMed: 18362358]

17. Raveche ES, Salerno E, Scaglione BJ, Manohar V, Abbasi F, Lin YC, et al. Abnormal microRNA-16 locus with synteny to human 13q14 linked to CLL in NZB mice. Blood 2007;109:5079-86. [PubMed: 17351108]

18. Ouillette P, Erba H, Kujawski L, Kaminski M, Shedden K, Malek SN. Integrated genomic profiling of chronic lymphocytic leukemia identifies subtypes of deletion 13q14. Cancer Res 2008;68:1012-21. [PubMed: 18281475]

19. Hammarsund M, Corcoran MM, Wilson W, Zhu C, Einhorn S, Sangfelt O, et al. Characterization of a novel B-CLL candidate gene--DLEU7--located in the 13q14 tumor suppressor locus. FEBS Lett 2004;556:75-80. [PubMed: 14706829]

20. Palamarchuk A, Efanov A, Nazaryan N, Santanam U, Alder H, Rassenti L, et al. 13q14 deletions in CLL involve cooperating tumor suppressors. Blood 115:3916-22. [PubMed: 20071661]

21. Pekarsky Y, Zanesi N, Aqeilan RI, Croce CM. Animal models for chronic lymphocytic leukemia. J Cell Biochem 2007;100:1109-18. [PubMed: 17131382]

22. Planelles L, Carvalho-Pinto CE, Hardenberg G, Smaniotto S, Savino W, Gomez-Caro R, et al. APRIL promotes B-1 cell-associated neoplasm. Cancer Cell 2004;6:399-408. [PubMed: 15488762]

23. Pekarsky Y, Palamarchuk A, Maximov V, Efanov A, Nazaryan N, Santanam U, et al. Tcl1 functions as a transcriptional regulator and is directly involved in the pathogenesis of CLL. Proc Natl Acad Sci U S A 2008;105:19643-8. [PubMed: 19064921]

24. Haiat S, Billard C, Quiney C, Ajchenbaum-Cymbalista F, Kolb JP. Role of BAFF and APRIL in human B-cell chronic lymphocytic leukaemia. Immunology 2006;118:281-92. [PubMed: 16827889]

25. Mackay F, Schneider P, Rennert P, Browning J. BAFF AND APRIL: a tutorial on B cell survival. Annu Rev Immunol 2003;21:231-64. [PubMed: 12427767]

26. Schuh K, Avots A, Tony HP, Serfling E, Kneitz C. Nuclear NF-ATp is a hallmark of unstimulated B cells from B-CLL patients. Leuk Lymphoma 1996;23:583-92. [PubMed: 9031090]

27. Berland R, Wortis HH. An NFAT-dependent enhancer is necessary for anti-IgM-mediated induction of murine CD5 expression in primary splenic B cells. J Immunol 1998;161:277-85. [PubMed: 9647234]

28. Virgilio L, Narducci MG, Isobe M, Billips LG, Cooper MD, Croce CM, et al. Identification of the TCL1 gene involved in T-cell malignancies. Proc Natl Acad Sci U S A 1994;91:12530-4. [PubMed: 7809072] 
29. Narducci MG, Pescarmona E, Lazzeri C, Signoretti S, Lavinia AM, Remotti D, et al. Regulation of TCL1 expression in B- and T-cell lymphomas and reactive lymphoid tissues. Cancer Res 2000;60:2095-100. [PubMed: 10786666]

30. Said JW, Hoyer KK, French SW, Rosenfelt L, Garcia-Lloret M, Koh PJ, et al. TCL1 oncogene expression in B cell subsets from lymphoid hyperplasia and distinct classes of B cell lymphoma. Lab Invest 2001;81:555-64. [PubMed: 11304575]

31. Bichi R, Shinton SA, Martin ES, Koval A, Calin GA, Cesari R, et al. Human chronic lymphocytic leukemia modeled in mouse by targeted TCL1 expression. Proc Natl Acad Sci U S A 2002;99:6955-60. [PubMed: 12011454]

32. Herling M, Patel KA, Khalili J, Schlette E, Kobayashi R, Medeiros LJ, et al. TCL1 shows a regulated expression pattern in chronic lymphocytic leukemia that correlates with molecular subtypes and proliferative state. Leukemia 2006;20:280-5. [PubMed: 16341048]

33. Pekarsky Y, Koval A, Hallas C, Bichi R, Tresini M, Malstrom S, et al. Tcl1 enhances Akt kinase activity and mediates its nuclear translocation. Proc Natl Acad Sci U S A 2000;97:3028-33. [PubMed: 10716693]

34. Laine J, Kunstle G, Obata T, Sha M, Noguchi M. The protooncogene TCL1 is an Akt kinase coactivator. Mol Cell 2000;6:395-407. [PubMed: 10983986]

35. Chan TO, Rittenhouse SE, Tsichlis PN. AKT/PKB and other D3 phosphoinositide-regulated kinases: kinase activation by phosphoinositide-dependent phosphorylation. Annu Rev Biochem 1999;68:965-1014. [PubMed: 10872470]

36. Pekarsky Y, Santanam U, Cimmino A, Palamarchuk A, Efanov A, Maximov V, et al. Tcl1 expression in chronic lymphocytic leukemia is regulated by miR-29 and miR-181. Cancer Res 2006;66:11590-3. [PubMed: 17178851]

37. Calin GA, Liu CG, Sevignani C, Ferracin M, Felli N, Dumitru CD, et al. MicroRNA profiling reveals distinct signatures in B cell chronic lymphocytic leukemias. Proc Natl Acad Sci U S A 2004;101:11755-60. [PubMed: 15284443]

38. Calin GA, Ferracin M, Cimmino A, Di Leva G, Shimizu M, Wojcik SE, et al. A MicroRNA signature associated with prognosis and progression in chronic lymphocytic leukemia. N Engl J Med 2005;353:1793-801. [PubMed: 16251535]

39. Malstrom S, Tili E, Kappes D, Ceci JD, Tsichlis PN. Tumor induction by an Lck-MyrAkt transgene is delayed by mechanisms controlling the size of the thymus. Proc Natl Acad Sci U S A 2001;98:14967-72. [PubMed: 11752445]

40. Suzuki A, Kaisho T, Ohishi M, Tsukio-Yamaguchi M, Tsubata T, Koni PA, et al. Critical roles of Pten in B cell homeostasis and immunoglobulin class switch recombination. J Exp Med 2003;197:657-67. [PubMed: 12615906]

41. Ghosh AK, Varga J. The transcriptional coactivator and acetyltransferase p300 in fibroblast biology and fibrosis. J Cell Physiol 2007;213:663-71. [PubMed: 17559085]

42. Hess J, Angel P, Schorpp-Kistner M. AP-1 subunits: quarrel and harmony among siblings. J Cell Sci 2004;117:5965-73. [PubMed: 15564374]

43. Leppa S, Bohmann D. Diverse functions of JNK signaling and c-Jun in stress response and apoptosis. Oncogene 1999;18:6158-62. [PubMed: 10557107]

44. Ameyar M, Wisniewska M, Weitzman JB. A role for AP-1 in apoptosis: the case for and against. Biochimie 2003;85:747-52. [PubMed: 14585541]

45. Foletta VC, Segal DH, Cohen DR. Transcriptional regulation in the immune system: all roads lead to AP-1. J Leukoc Biol 1998;63:139-52. [PubMed: 9468273]

46. Colotta F, Polentarutti N, Sironi M, Mantovani A. Expression and involvement of c-fos and c-jun protooncogenes in programmed cell death induced by growth factor deprivation in lymphoid cell lines. J Biol Chem 1992;267:18278-83. [PubMed: 1526968]

47. Ott RG, Simma O, Kollmann K, Weisz E, Zebedin EM, Schorpp-Kistner M, et al. JunB is a gatekeeper for B-lymphoid leukemia. Oncogene 2007;26:4863-71. [PubMed: 17297445]

48. Kantor AB, Herzenberg LA. Origin of murine B cell lineages. Annu Rev Immunol 1993;11:50138. [PubMed: 8476571] 
49. Hoyer KK, French SW, Turner DE, Nguyen MT, Renard M, Malone CS, et al. Dysregulated TCL1 promotes multiple classes of mature B cell lymphoma. Proc Natl Acad Sci U S A 2002;99:143927. [PubMed: 12381789]

50. Zanesi N, Aqeilan R, Drusco A, Kaou M, Sevignani C, Costinean S, et al. Effect of Rapamycin on Mouse Chronic Lymphocytic Leukemia and the Development of Nonhematopoietic Malignancies in Em-TCL1 Transgenic Mice. Cancer Res 2006;66:915-20. [PubMed: 16424025]

51. Robak T. Second malignancies and Richter's syndrome in patients with chronic lymphocytic leukemia. Hematology 2004;9:387-400. [PubMed: 15763979]

52. Kyasa MJ, Hazlett L, Parrish RS, Schichman SA, Zent CS. Veterans with chronic lymphocytic leukemia/small lymphocytic lymphoma (CLL/SLL) have a markedly increased rate of second malignancy, which is the most common cause of death. Leuk Lymphoma 2004;45:507-13. [PubMed: 15160912]

53. Parekh K, Rusch V, Kris M. The clinical course of lung carcinoma in patients with chronic lymphocytic leukemia. Cancer 1999;86:1720-3. [PubMed: 10547544]

54. Efanov A, Zanesi N, Nazaryan N, Santanam U, Palamarchuk A, Croce CM, et al. CD5+CD23+ leukemic cell populations in TCL1 transgenic mice show significantly increased proliferation and Akt phosphorylation. Leukemia 2010;24:970-975. [PubMed: 20357824]

55. Santanam U, Zanesi N, Efanov A, Costinean S, Palamarchuk A, Hagan JP, et al. Chronic lymphocytic leukemia modeled in mouse by targeted miR-29 expression. Proc Natl Acad Sci U S A 2010;107:12210-12215. [PubMed: 20566844]

56. Zanesi N, Pekarsky Y, Trapasso F, Calin G, Croce C. MicroRNAs in mouse models of lymphoid malignancies. J Nucleic Acids Invest 2010;1:36-40.

57. Klein U, Lia M, Crespo M, Siegel R, Shen Q, Mo T, et al. The DLEU2/miR-15a/16-1 cluster controls B cell proliferation and its deletion leads to chronic lymphocytic leukemia. Cancer Cell 17:28-40. [PubMed: 20060366]

58. Liu Y, Corcoran M, Rasool O, Ivanova G, Ibbotson R, Grander D, et al. Cloning of two candidate tumor suppressor genes within a $10 \mathrm{~kb}$ region on chromosome $13 \mathrm{q} 14$, frequently deleted in chronic lymphocytic leukemia. Oncogene 1997;15:2463-73. [PubMed: 9395242]

59. Zapata JM, Krajewska M, Morse HC 3rd, Choi Y, Reed JC. TNF receptor-associated factor (TRAF) domain and Bcl-2 cooperate to induce small B cell lymphoma/chronic lymphocytic leukemia in transgenic mice. Proc Natl Acad Sci U S A 2004;101:16600-5. [PubMed: 15545599]

60. Katsumata M, Siegel RM, Louie DC, Miyashita T, Tsujimoto Y, Nowell PC, et al. Differential effects of Bcl-2 on T and B cells in transgenic mice. Proc Natl Acad Sci U S A 1992;89:11376-80. [PubMed: 1454823]

61. Lee SY, Reichlin A, Santana A, Sokol KA, Nussenzweig MC, Choi Y. TRAF2 is essential for JNK but not NF-kappaB activation and regulates lymphocyte proliferation and survival. Immunity 1997;7:703-13. [PubMed: 9390693]

62. Chung JY, Park YC, Ye H, Wu H. All TRAFs are not created equal: common and distinct molecular mechanisms of TRAF-mediated signal transduction. J Cell Sci 2002;115:679-88. [PubMed: 11865024] 


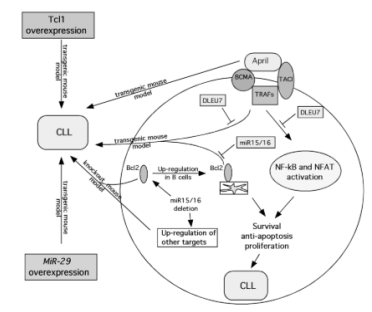

Figure 1.

Schematic representation of molecular pathways in CLL and animal models of CLL. 


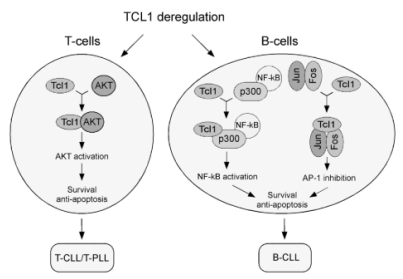

Figure 2.

Schematic representation of Tcl1 oncogenic function in T-cells and in B-cells. 